\title{
Efficient Database Encryption Scheme for Database-as-a-Service Environment
}

\author{
Hankyu Joo \\ Hallym University \\ hkjoo@hallym.ac.kr
}

\begin{abstract}
Computing-as-a-service is gaining ground. Clients may use the service without purchasing the system supporting the service. Database-as-a-Service (DBaaS) is an important area of computing-as-a-service. DBaaS allows clients to use an expensive database management system without purchasing it. In a DBaaS environment, database Tables are stored on servers belonging to a service provider, and hence, they must be encrypted in order to ensure data confidentiality. However, the encryption introduces performance degradation in the execution of queries over encrypted data. The execution of range queries, in particular, undergoes severe performance degradation. Several encryption schemes to alleviate this problem have been proposed. However, most of these schemes leak other information in addition to order information. In this study, a new database encryption scheme for DBaaS is proposed. The proposed scheme enables the execution of range queries without severe performance degradation and without leakage of information other than order information.
\end{abstract}

Keywords: database-as-a-service, confidentiality, database encryption, order preserving encryption

\section{Introduction}

Nowadays, computing as a service is gaining ground. Clients may use the service without purchasing the system supporting the service. One of the areas in computing as a service is Database-as-a-service (DBaaS). With DBaaS, clients may use an expensive database management system without purchasing the system [1].

In DBaaS, clients store their data Tables on servers that belong to the service providers. These servers are not under the control of clients, and hence, there is no guarantee of confidentiality of the client data that is stored on the servers without a proper protection scheme. Clients may encrypt the sensitive data to ensure data confidentiality. However, the encryption of data introduces performance degradation in the execution of queries over encrypted data.

When a column of a database Table is encrypted, exact queries can be executed without severe performance degradation by maintaining the index on the encrypted column. The client may encrypt the search condition and send a query of the encrypted condition to the server.

However, the execution of range queries undergoes severe performance degradation. In order to execute a range query, the server must decrypt each encrypted item to determine whether the item is in the range. The index is maintained on the encrypted data, and hence, it may not be used for performance enhancement.

Several approaches to alleviate the performance degradation have been proposed. In order to alleviate the problem, the server must be able to perform the order comparison without decrypting the encrypted items. In order to enable the server to perform order comparison without decryption, Order-Preserving Encryption (OPE) schemes have been proposed. Some of the proposed OPE schemes allow servers to perform an order 
comparison without decryption; however, they leak other information in addition to order information. Other schemes require the server to perform operations that are not database operations. The server must support such operations.

In this study, a database encryption scheme that does not result in severe performance degradation is proposed. The proposed scheme leaks only order information and uses only database operations of the server.

The remainder of this paper is organized as follows: Related work is discussed in Section 2. Our proposed database encryption scheme is described in Section 3. The proposed scheme is evaluated in Section 4. The conclusion of the study is presented in Section 5.

\section{Related Work}

A large amount of research has been conducted in the field of database encryption. Database encryption has encountered severe performance degradation in the execution of range queries. Research in database encryption has mainly focused on alleviating performance degradation. OPE was proposed for database encryption by Agrawal, et al., [2] to alleviate performance degradation. Boldyreva, et al., [3] proposed a new security definition for order-preserving encryption called indistinguishability under ordered chosen-plaintext attack (IND-OCPA). The introduction of OPE by [2] was followed by numerous studies in the field [3-6]. OPE allows the encryption function to preserve the numerical ordering of the plaintexts. Another similar scheme called order-preserving encoding has also been proposed [7]. An order-preserving encoding scheme maintains tree-structured order information on the server.

Studies such as [7-9] indicate that most of the proposed OPE schemes leak other information in addition to order information. Another scheme [7] requires operations that are not database operations to be performed at the server. Joo [10] proposed a basic approach to the database encryption scheme in which an order column is added for each encrypted column. According to Kolesnikov and Shikfa [11], even an ideal encryption system with order information leaks other information.

\section{Proposed Scheme}

In this section, the proposed database encryption scheme is described. In Section 3.1, the assumptions on which the design of the scheme is based are listed. The encryption scheme is described in Section 3.2. The algorithm for range query is presented in Section 3.3 and the algorithm for insertion is discussed in Section 3.4.

\subsection{Assumptions}

The design of a new scheme is based on the following assumptions:

- In DBaaS, all database Tables, including those containing confidential information, are stored on the server of the service provider. The server is a database server, and hence, it can be accessed only by database operation interfaces.

- The cost of storage is not high. This implies that an increase in the Table size does not result in excessive cost.

- In many applications, database Tables are initialized with a large number of items. After the initialization, query operations are extremely frequent. The insertion of items is required; however, it is not a frequent operation. Extensive Table modification may occur periodically.

- Indexing is used to improve the query speed. 


\subsection{Database Encryption Scheme}

The proposed scheme uses an extended Table. When a Table is created and stored on a server, each column having confidential data $(x)$ of a database Table is encrypted and renamed $(e X)$, and an additional order column $(o X)$ is added. The encryption may be performed by using any existing secure encryption algorithm such as Advanced Encryption Standard (AES) [12]. The order column $(o X)$ is used to maintain the order of the confidential data column $(x)$. Let us assume that an item $m$ has a value $x_{m}$ in column $x$ and $o X_{m}$ in column $o X$. Further, an item $n$ has a value $x_{n}$ in column $x$ and $o X_{n}$ in column $o X$. Then, if $x_{m}<x_{n}, o X_{m}<o X_{n}$.

For example, let us consider an emp Table, Table 1. The Table contains four columns: ID, Name, Title, and Salary. The columns, ID and Salary, contain confidential information.

Table 1. Plaintext emp Table

\begin{tabular}{lllc}
\hline ID & Name & Title & Salary \\
\hline $650213-1234567$ & Hankyu Joo & Manager & 14,000 \\
$690313-2345678$ & Sangmin Han & Programmer & 12,000 \\
$700225-1234567$ & Jaewook Choi & Programmer & 15,000 \\
$\ldots$. & $\ldots$ & $\ldots$ & $\ldots$ \\
\hline
\end{tabular}

In order to ensure security, the data in the columns, $I D$ and Salary, are encrypted. The corresponding column names are renamed as eID and eSalary, as shown in Table 2. The columns, $o I D$ and $o$ Salary, with order information of $I D$ and Salary, respectively, are also added. The extended Table is named eEmp and is stored on the server.

Table 2. Encrypted eEmp Table

\begin{tabular}{lllccl}
\hline eID & Name & Title & eSalary & oID & oSalary \\
\hline eX12Klm & Hankyu Joo & Manager & tyK3xDs & 200 & 600 \\
jMcD38h & Sangmin Han & Programmer & wQ1b01d & 700 & 300 \\
y08b1xK & Jaewook Choi & Programmer & d2IpRz9 & 900 & 900 \\
$\ldots$. & $\ldots$. & $\ldots$ & $\ldots$ & $\ldots$ & $\ldots$ \\
\hline
\end{tabular}

Indexes are created for the columns, eID, eSalary, oID, and oSalary.

The encryption and order calculation is performed on the computer of the client. When a Table is created, the order column has a large interval. Although $x_{n}$ and $x_{m}$ are consecutive in order, $o X_{n .}-o X_{m}$ is not one but a large number such as 100 . Indexes are used whenever necessary.

\subsection{Range Query for Encrypted Tables}

For unencrypted database Tables, the following range query may be performed. The column $x$ may be indexed to improve the query speed.

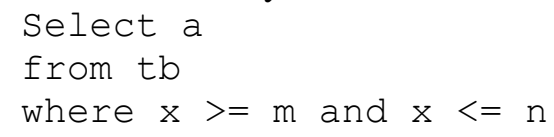

If the column $x$ contains confidential information, this column is encrypted as the column $e X$ and the encrypted Table is renamed as $e T b$. In the encrypted Table, the same format of range query results in severe performance degradation. In order to alleviate the performance degradation, the query should be changed as follows: 


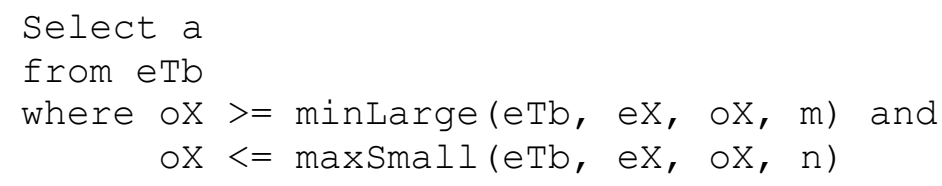

For example, the following query may be executed to obtain the names corresponding to a salary between 12,000 and 15,000 in Table 1. The column Salary may be indexed to improve the query speed.

Select Name

from employee

where Salary $>=12,000$ and Salary $<=15,000$

If the column Salary is encrypted as the column eSalary, and if the column oSalary maintains the order of Salary as seen in Table 2, the query should be modified as follows.

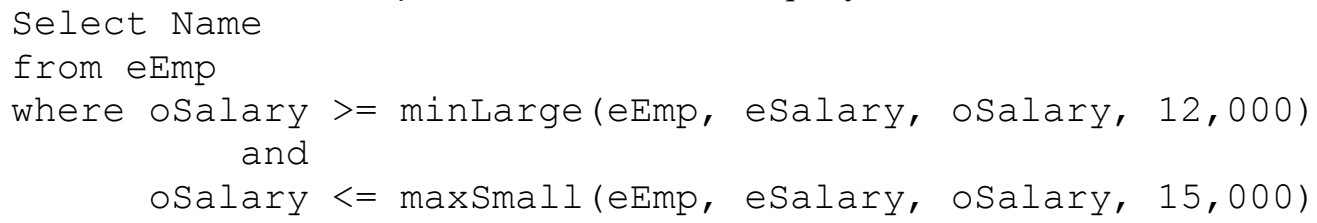

The functions minLarge and maxSmall are executed on the computer of the client. The client communicates with the server to obtain the necessary information by using only the query select. The function minLarge(eTable, field, order, value) is used to obtain the order of an item whose decrypted field has the least value greater than or equal to value in eTable. The algorithm for function minLarge(eTable, field, order, value) is described in Table 3. The function maxSmall(eTable, field, order, value) is used to obtain the order of an item whose decrypted field has the largest value smaller than or equal to value in eTable. The algorithm for function maxSmall(eTable, field, order, value) is described in Table 4.

Table 3. MinLarge Algorithm

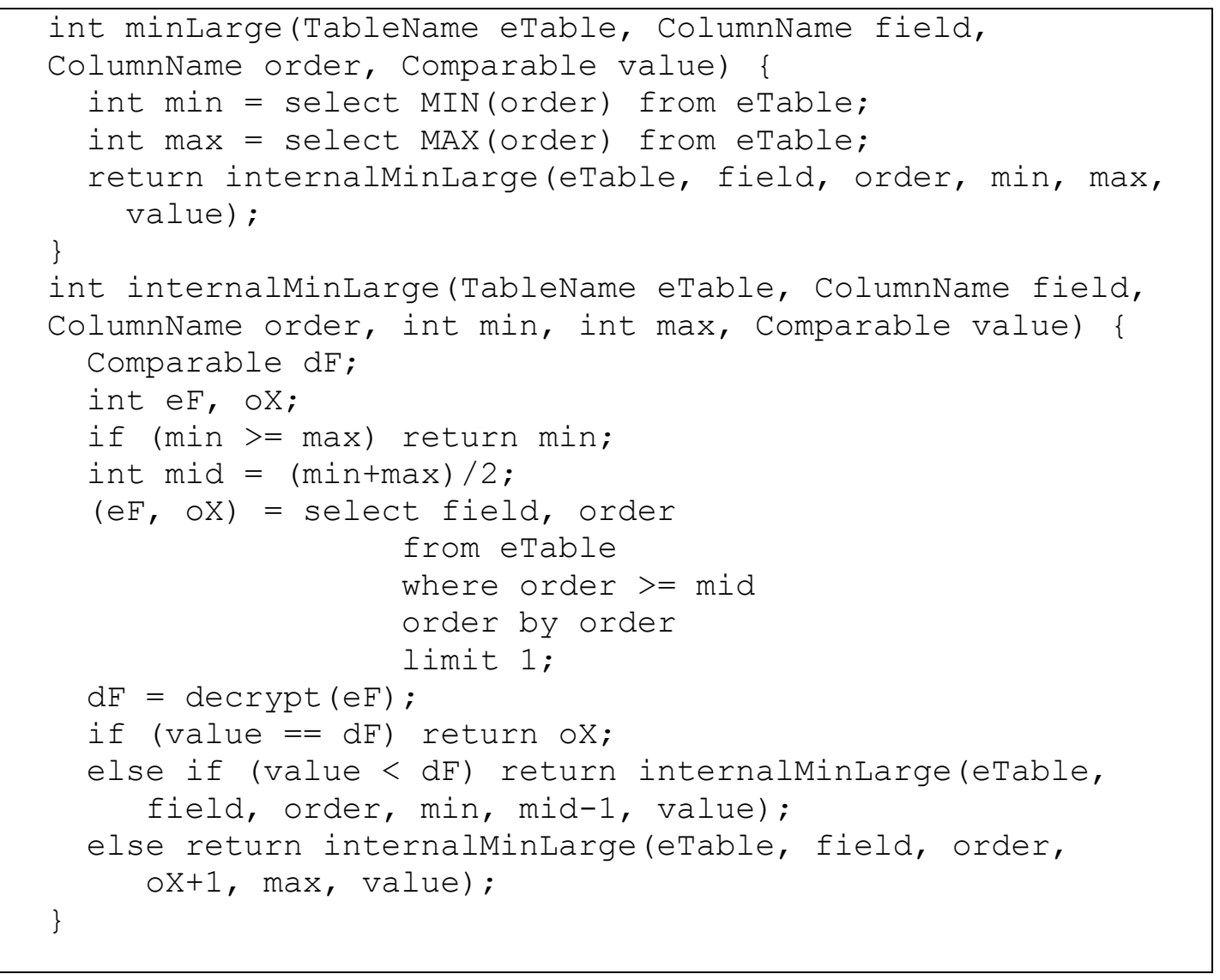


The function minLarge obtains the minimum ( $\min$ ) and maximum (max) values in the column order of eTable. With these values, minLarge calls the function internalMinLarge. The function internalMinLarge(eTable, field, order, min, max, value) is a recursive function to determine the order of an item whose decrypted field has the least value greater than or equal to value. The internalMinLarge(eTable, field, order, min, max, value) searches only those items whose order is between min and max. When internalMinLarge is called, an item whose column order has the least value greater than or equal to mid is selected from eTable. The selected item is checked to determine whether its decrypted field is the same as value. If the decrypted field of the item is the same as value, the order of the item is found and returned. If the decrypted field is smaller than value, internalMinLarge is called with the range between min and mid. If the decrypted field is larger than value, internalMinLarge is called with the range between mid and max. After successive recursive calls, min becomes greater than or equal to max. At that point, $\min$ has the order of the item whose field is least greater than value.

\section{Table 4. MaxSmall Algorithm}

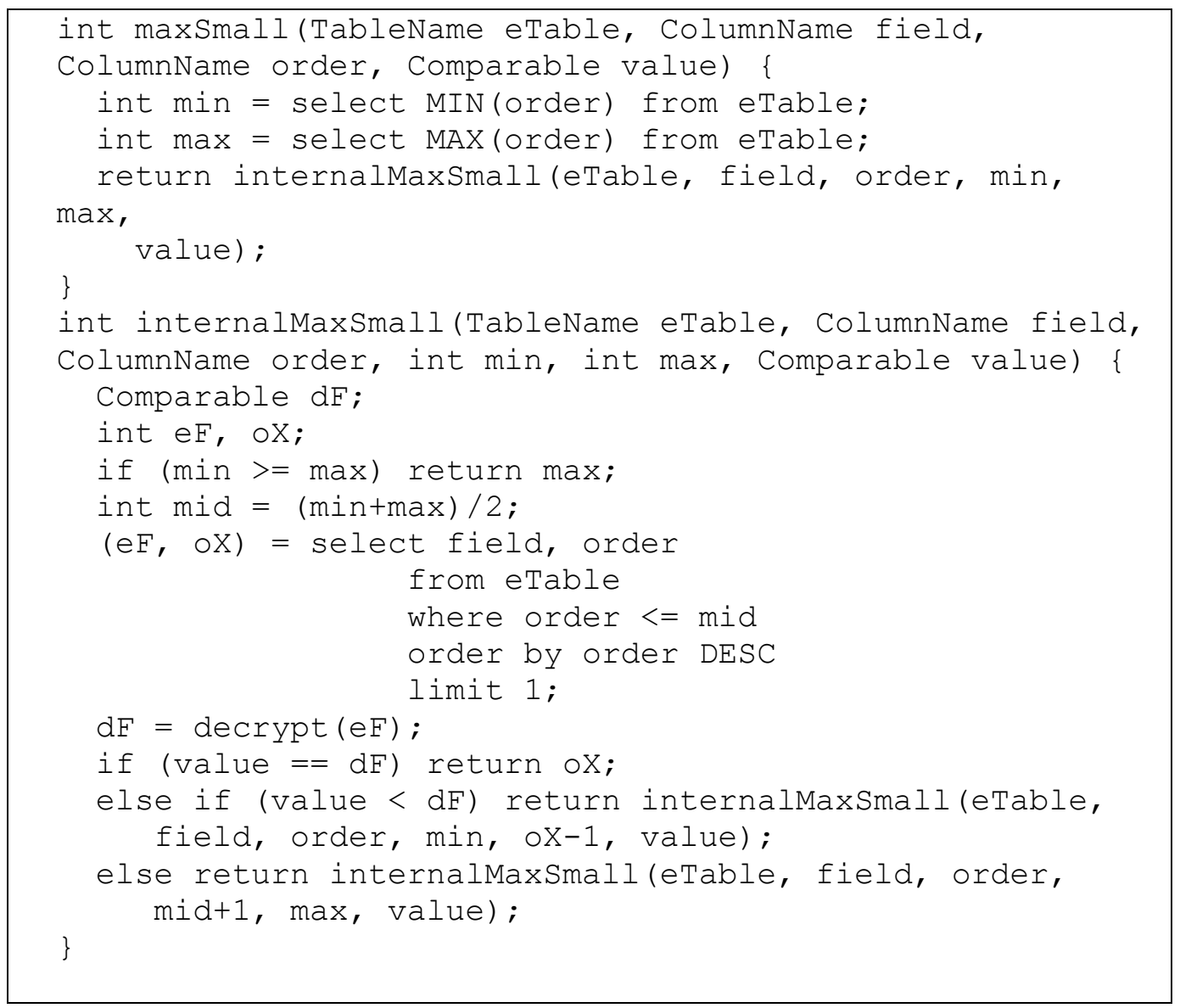

The function maxSmall works in a similar manner as the function minLarge. The function maxSmall obtains the minimum ( $\min$ ) and maximum ( $\max$ ) values in the column order of eTable. With these values, maxSmall calls the function internalMaxSmall. The function internalMaxSmall(eTable, field, order, $\min , \max$, value) is a recursive function to determine the order of an item whose decrypted field has the largest value smaller than or equal to value. The internalMaxSmall( eTable, field, order, min, max, value) searches only those items whose order is between min and max. When internalMaxSmall is called, an item whose column order has the largest value smaller than or equal to mid is selected 
from $e$ Table. The selected item is checked to determine whether its decrypted field is the same as value. If the decrypted field of the item is the same as value, the order of the item is found and returned. If the decrypted field is smaller than value, internalMaxSmall is called with the range between min and mid. If the decrypted field is larger than value, internalMaxSmall is called with the range between mid and max. After successive recursive calls, min becomes greater than or equal to max. At that point, max is the order of the item whose field has the largest value smaller than value.

\subsection{Insertion into Encrypted Tables}

When a new item is inserted into an unencrypted database Table, the following insert statement must be executed, where $T b$ is the Table name, $a$ and $x$ are column names, and $a V$ and $x V$ are values corresponding to the columns, $a$ and $x$ :

Insert Into Tb $(a, x)$

Values $(\mathrm{aV}, \mathrm{xV})$;

When a new item is inserted into an encrypted Table, a value for the column order must be calculated. The value of the column order for the new item is the average value of the two neighboring order values. Let us assume that the encrypted Table is $e T b, e X$ is the encrypted column corresponding to $x$, and $o X$ is the column order corresponding to $x$. The following insert statement must be executed:

Insert Into eTb (a, eX, oX)

Values (aV, encrypt (xV), newOx (eTb, eX, oX, $x V))$;

The algorithm newOx in Table 5 is used to calculate a new value of order corresponding to the value that is supposed to be encrypted. The function newOx is executed on the computer of the client. The client communicates with the server to obtain the necessary information by using only the query select.

\section{Table 5. NewOx Algorithm}

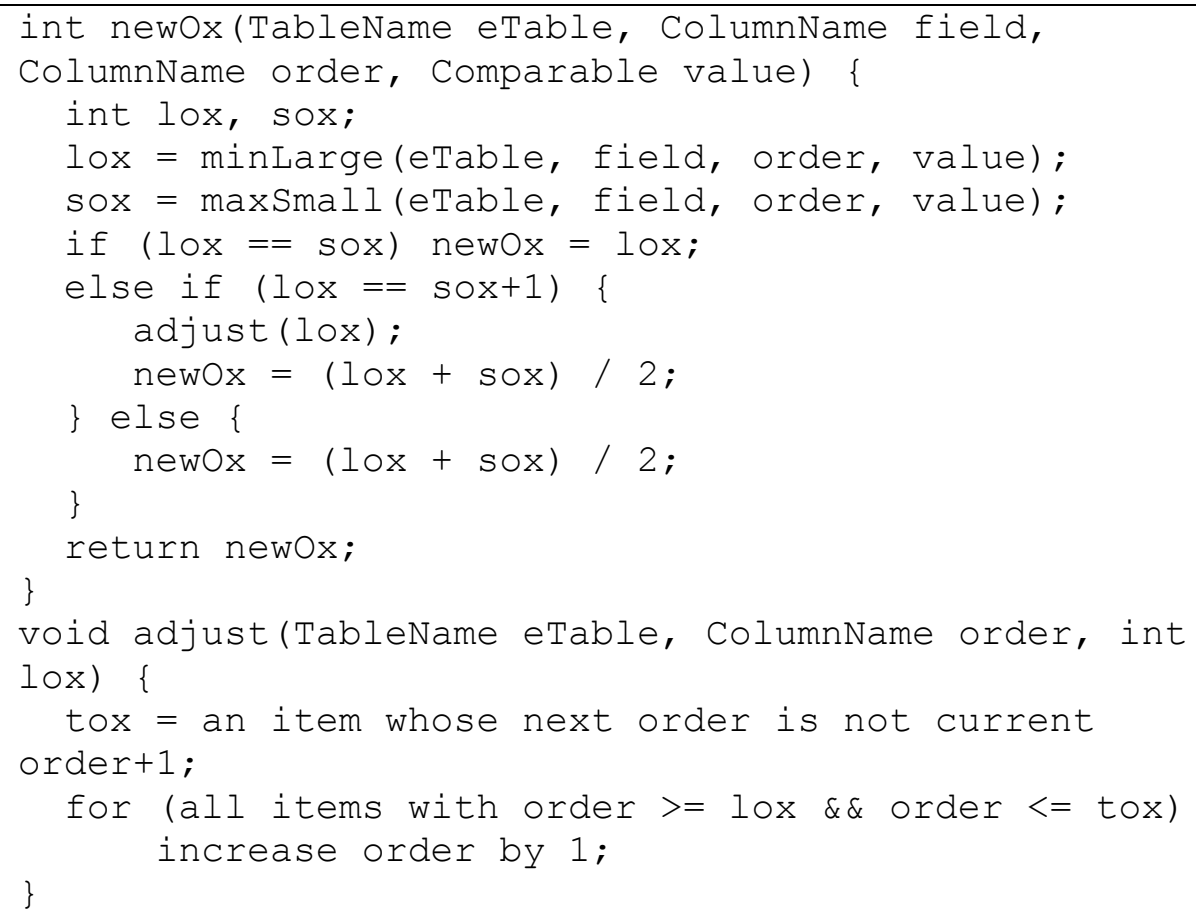

The function newOx(eTable, field, order, value) is used to calculate the new value of order corresponding to the value that is supposed to be encrypted. The function new $O x$ 
uses minLarge and maxSmall functions that are described in Tables 3 and 4, respectively. The order of the item, which has the smallest value of order that is larger than or equal to the new item with value, is selected from eTable and saved as lox. The order of the item, which has the largest value of order that is smaller than or equal to the new item with value, is selected from eTable and saved as sox. The new order value (newOx) is the average value of lox and sox. The function adjust is called when it is not possible to have a new order because the existing order interval is 1 .

If multiple order columns are added, the number of times that the function newOx is called is equal to the number of columns added.

For example, let us suppose that an item (690812-2011369, Kyoung Kim, Secretary, 12,500) must be added to Table 1. In order to insert the item, the following insert statement must be executed.

Insert Into emp (ID, Name, Title, Salary)

Values (690812-2011369, Kyoung Kim, Secretary, 12,500);

After the insertion, the Table emp is modified as shown in Table 6.

Table 6. Modified emp Table

\begin{tabular}{lllc}
\hline ID & Name & Title & Salary \\
\hline $650213-1234567$ & Hankyu Joo & Manager & 14,000 \\
$690313-2345678$ & Sangmin Han & Programmer & 12,000 \\
$690812-2011369$ & Kyoung Kim & Secretary & 12,500 \\
$700225-1234567$ & Jaewook Choi & Programmer & 15,000 \\
$\ldots$. & $\ldots$. & $\ldots$. & $\ldots$ \\
\hline
\end{tabular}

In order to insert the item into an encrypted Table as shown in Table 2, the following insert statement must be executed.

Insert Into eEmp (eID, Name, Title, eSalary, oId, oSalary)

Values (encrypt(690812-2011369), Kyoung Kim, Secretary, encrypt (12,500), newOx(eEmp, eID, OID, 690812-2011369), newOx (eEmp, eSalary, oSalary, 12,500));

If the above insert statement is executed, an item (Dj8L1s, Kyoung Kim, Secretary, $q 9 P \ln T, 800,450$ ) will be added to Table 2, thus generating Table 7.

Table 7. Modified eEmp Table

\begin{tabular}{|c|c|c|c|c|c|}
\hline eID & Name & Title & eSalary & oID & oSalar \\
\hline eX12Klm & Hankyu Joo & Manager & tyK3xDs & 200 & 600 \\
\hline jMcD38h & Sangmin Han & Programme & wQ1b01d & 700 & 300 \\
\hline Dj8L1s & Kyoung Kim & Secretary & q9PlnT & 800 & 450 \\
\hline y08b $1 x K$ & Jaewook Choi & Programme & $\mathrm{d} 2 \mathrm{IpRz} 9$ & 900 & 900 \\
\hline$\ldots$ & $\ldots$ & $\ldots$ & $\ldots$ & $\ldots$ & $\ldots$ \\
\hline
\end{tabular}

Periodically, a large number of items may be modified. When this occurs, the Table may be retrieved to the client side, and the order columns may be recalculated by using the same approach as used in Table initialization. 


\section{Evaluation}

The encryption of a column uses an existing secure encryption algorithm, and hence, the proposed scheme leaks only order information. This scheme uses only standard query operations to access the server and does not require any changes at the server. In the absence of order information, the performance of a range query for an encrypted column is $\mathrm{O}(n)$ slower than that for a plaintext column, where $n$ is the total number of items in the Table. In the proposed scheme, the addition of the order column to the encrypted columns results in the performance of the range query for the encrypted column to be $\mathrm{O}\left(\log _{n}\right)$ slower than that for a plaintext column. In the proposed method, the insertion operation may be performed in $\mathrm{O}\left(\log _{n}\right)$. If many items are inserted for a specific range of values, the order column may be restructured. By inspecting the order column, clients may learn about its status.

\section{Conclusion}

In this study, a new scheme for database encryption is proposed. The proposed scheme may be implemented only on the client side without any changes to the server. It does not leak any information except the order of the encrypted field. The proposed method may execute a range query on the encrypted column without resulting in severe performance degradation. The proposed scheme requires additional columns in the encrypted Table. However, the additional storage for these columns is not expensive in a DBaaS environment. In this method, periodic inspection of the order column is necessary and possible restructuring of the column may be required. However, database insertion is not a frequent operation and restructuring may only be necessary when the original Table undergoes a major update.

\section{ACKNOWLEDGEMENTS}

This research was supported by Hallym University Research Fund, 201504 (HRF201504-012).

\section{References}

[1] H. Hacigumus, B. Iyer and S. Mehrotra, "Providing database as a service," Proceedings of 18th International Conference on Data Engineering, (2002) February 26-March 1, San Jose, California.

[2] R. Agrawal, J. Kiernan, R. Srikant and Y. Xu, "Order preserving encryption for numeric data," ACM SIGMOD, (2004), pp. 563-574.

[3] A. Boldyreva, N. Chenette, Y. Lee and A. O'Neill, "Order-preserving symmetric encryption," EUROCRYPT, LNCS 5479. (2009), pp. 224-241.

[4] A. Boldyreva, N. Chenette and A. O'Neill, "Order-preserving symmetric encryption revisited: Improved security analysis and alternative solutions," CRYPTO 2011, LNCS 6841, (2011), pp. 578-595.

[5] D. H. Yum, D. S. Kim, J. S. Kim, P. J. Lee and S. J. Hong, "Order-preserving encryption for nonuniformly distributed plaintexts," WISA 2011, LNCS 7115, (2012), pp. 84-97.

[6] D. Liu and S. Wang, "Nonlinear order preserving index for encrypted database query in service cloud environments," Concurrency and Computation: Practice and Experience, vol. 25, (2013), pp. 1967-1984.

[7] R. A. Popa, F. H. Li and N. Zeldovich, "An ideal-security protocol for order-preserving encoding," IEEE Symposium on Security and Privacy, (2013), pp. 463-477.

[8] X. Liangliang, O. Bastani and I. Yen, "Security Analysis for an Order-Preserving Schemes," Technical Report UTDCS-06-10, University of Texas at Dallas, (2010).

[9] X. Liangliang, I. Yen and D. Lin, "Security Analysis for an Order-Preserving Schemes," Technical Report UTDCS-01-12, University of Texas at Dallas, (2012).

[10] H. Joo, "Practical Database Encryption Scheme for Database-as-a-Service," Advanced Science and Technology Letters, Security, Reliability and Safety, vol. 93, (2015), pp. 34-39.

[11] V. Kolesnikov and A. Shikfa, "On The Limits of Privacy Provided by Order-Preserving Encryption," Bell Labs Technical Journal, vol. 17, no. 3, (2012), pp. 135-146.

[12] National Institute of Standards and Technology, "Advanced Encryption Standard (AES)," FIPS PUB, vol. 197, (2001). 


\begin{abstract}
Author
Hankyu Joo, received his B.Sc. degree in Computer Science from Hallym University, Korea, in 1988. He received his M.S. and Ph.D. degrees in Computer Science and Engineering from Arizona State University in 1994 and 1998, respectively. He worked for the Electronics and Telecommunications Research Institute, Korea, from 1999 to 2000. He is a professor of Computer Engineering at Hallym University. His research interests include information security and software engineering.
\end{abstract}


International Journal of Security and Its Applications Vol. 9, No. 5 (2015) 